\title{
Utilization of biodegradable polymeric materials as delivery agents in dermatology
}

This article was published in the following Dove Press journal:

Clinical, Cosmetic and Investigational Dermatology

9 January 2014

Number of times this article has been viewed

Fiorenza Rancan

Ulrike Blume-Peytavi

Annika Vogt

Clinical Research Center for Hair and Skin Science, Department of

Dermatology and Allergy, Charité -

Universitätsmedizin Berlin, Germany
Abstract: Biodegradable polymeric materials are ideal carrier systems for biomedical applications. Features like controlled and sustained delivery, improved drug pharmacokinetics, reduced side effects and safe degradation make the use of these materials very attractive in a lot of medical fields, with dermatology included. A number of studies have shown that particlebased formulations can improve the skin penetration of topically applied drugs. However, for a successful translation of these promising results into a clinical application, a more rational approach is needed to take into account the different properties of diseased skin and the fate of these polymeric materials after topical application. In fact, each pathological skin condition poses different challenges and the way diseased skin interacts with polymeric carriers might be markedly different to that of healthy skin. In most inflammatory skin conditions, the skin's barrier is impaired and the local immune system is activated. A better understanding of such mechanisms has the potential to improve the efficacy of carrier-based dermatotherapy. Such knowledge would allow the informed choice of the type of polymeric carrier depending on the skin condition to be treated, the type of drug to be loaded, and the desired release kinetics. Furthermore, a better control of polymer degradation and release properties in accordance with the skin environment would improve the safety and the selectivity of drug release. This review aims at summarizing the current knowledge on how polymeric delivery systems interact with healthy and diseased skin, giving an overview of the challenges that different pathological skin conditions pose to the development of safer and more specific dermatotherapies.

Keywords: nanocarriers, nanoparticles, biodegradable polymers, skin penetration, hair follicles

\section{Introduction}

Nanotechnology offers a vast possibility to improve delivery, bioavailability, and targeting of drugs and cosmetics to skin. Considering the good accessibility of skin, the possibility to treat skin disorders by topical therapies is very appealing. This method of administration increases patient compliance, reduces side effects, avoids the hepatic first pass metabolism, hereby enhancing the amount of drug at the site of action, and could help to reduce the dose required to obtain therapeutic effects. ${ }^{1}$ Supramolecular drug delivery systems (DDS) applied topically to diseased skin should accumulate and release the drug at the site of action. In diseased skin, because of impaired stratum corneum (SC) and/or activated immune system, the penetration of macromolecules and particulate materials is often altered with respect to healthy skin. Thus, to avoid possible side effects, the use and development of biodegradable and biocompatible nanomaterials is necessary. In medicine, the term biodegradable describes materials
Correspondence: Fiorenza Rancan Clinical Research Center for Hair and Skin Science, Department of Dermatology and Allergy, Charité - Universitätsmedizin Berlin, Chariteplatz I, I0II7 Berlin, Germany Tel +4930450518347 Fax +4930450518952 Email fiorenza.rancan@charite.de 
which breakdown into molecules that are nontoxic and can be metabolized or eliminated by the body. The term biocompatible is often used to describe nondegradable materials that are eliminated by renal clearance and have been shown to be safe in in vivo studies. In this review, focus is given to biodegradable polymer materials that have been developed and tested as delivery agents for dermatotherapies. Some of the most investigated biocompatible polymeric materials will be also discussed. We aim to offer an overview of the different types of DDS and the key processes occurring after their topical administration. In particular, the different pathophysiological conditions occurring in skin diseases have been outlined in order to emphasize that each different pathological condition brings specific challenges that have to be taken into account when developing and investigating new carrier systems.

\section{Types of biodegradable polymeric DDS}

The first delivery systems that were investigated and marketed for dermatological and cosmetic applications were liposomes. ${ }^{2}$ Thereafter, solid lipid nanoparticles (SLN) and nanostructured lipid carriers (NLC) were developed, which are more stable and have better skin penetration. ${ }^{3}$ In the past few years, an increasing number of polymeric carriers have been investigated for topical drug delivery. ${ }^{4}$ Biodegradable polymers offer certain advantages over lipid-based carriers. They can be stored over months, both hydrophobic and hydrophilic drugs can be encapsulated or adsorbed, they can be functionalized, and most importantly their physicochemical properties can be adjusted in order to achieve a controlled release of the encapsulated drug. Several different types of polymer-based DDS have been tested for drug delivery. Polymeric fibers are used in tissue engineering as scaffolds for tissue reconstruction and wound healing. 5,6 Poly( $\varepsilon$-caprolactone) (PCL), collagen, and hyaluronic acid are some of the polymers used to prepare biological matrixes. They can be loaded with growth or angiogenic factors to favor tissue regrowth or antimicrobials to avoid wound infection. ${ }^{7,8}$ Hydrogels consist of hydrophilic molecules, called gelling agents, which are chemically or physically crosslinked and form a three-dimensional network with the ability to absorb high amounts of water. ${ }^{9}$ Hydrogels can be made of natural polymers, like alginate, chitosan, dextran, or of synthetic material like poly(ethylene glycol) (PEG), poly(lacticco-glycolic acid) (PLGA), or a combination of natural and synthetic polymers. ${ }^{10}$ They can be loaded with a variety of drugs, mainly hydrophilic ones, and have been largely investigated for drug delivery in medicine. Organogels are gelling agent networks able to incorporate oil or organic solvents. Polymers like poly(ethylene) and PEG, as well as copolymers of methacrylic acid ${ }^{11}$ and methyl methacrylate (MMA) are commonly used. These polymers are nonbiodegradable but are considered biocompatible; ${ }^{12}$ because of their lipophilic nature they can incorporate hydrophobic drugs and have a better skin penetration. ${ }^{13}$ Hydrogels have also been used to disperse nanocarriers enhancing their distribution on the skin surface and were applied as a delivery system for wound healing. ${ }^{14,15}$ Nanogels are nanoscaled organogels and hydrogels. Using appropriate linkers it is possible to make nanogels stimulus-responsive (eg, temperature, $\mathrm{pH}$, light). ${ }^{16,17}$ Thus, the release of encapsulated drugs can be controlled by exploiting the unique parameters of diseased skin or even using an external trigger. The small size and the deformability of nanogels favor their accumulation in the $\mathrm{SC}$ and in the hair follicle canal. Polymeric spheres are made of several polymer chains which in water spontaneously "roll-up" to form spherical particles. Both natural and synthetic polymers have been used. Natural polymers are derived from animals as well as plants and include polysaccharides, such as cellulose, chitin, starch, alginate, galactan, hyaluronic acid, dextran, and gellan, ${ }^{18}$ or protein-based carriers like albumin, gelatine, collagen, milk and silk proteins, soy proteins, and lectins. ${ }^{19,20}$ Polydepsipeptides are synthetic peptide polymers which are degraded into biocompatible amino acids. ${ }^{21}$ Their composition can be adjusted to achieve an optimal complexation of the loaded drug and to control their physicochemical properties such as hydrophobicity/ hydrophilicity. The most studied synthetic biodegradable polymers used to prepare micro and submicron spheres for drug delivery are poly(lactic acid) (PLA), ${ }^{22}$ PLGA and PCL. ${ }^{23,24}$ PLA particles are prepared from renewable sources. They allow for an easy-scalable and low cost production and are degraded to lactic acid, a resorbable and non-toxic monomer. ${ }^{25}$ Polymeric micelles are auto-assembled micelles made of amphiphilic macromolecules such as di- or triblock copolymers. Examples of biodegradable micelles are those based on PEG, PLA, PCL, or chitosan, whereas poly(amidoamine) (PAMAM) micelles are considered biocompatible. The advantages of such particles are their small size, the solubilization of hydrophobic drugs, and the possibility to functionalize their surface with targeting units. ${ }^{26}$ Dendrimers and dendritic polymers consist as macromolecules with a branched structure in which different types of drugs can be intercalated or covalently bound. Dendritic core-multishell nanocarriers have amphiphilic architecture 
consisting of a hydrophobic inner shell and a hydrophilic outer shell allowing the encapsulation of lipophilic drugs and the control of surface polarity. Polyester dendrimers made of glycerol, succinic acid, phenylalanine, and lactic acid are promising biodegradable carriers. ${ }^{27}$

Polymeric carriers are available in different sizes ranging from nanometer to submicron and micrometer dimension. In general, the organic nature of the polymer and the threedimensional conformation confer them flexibility and deformability, which are important requisites for penetration across tissue barriers.

\section{Key processes after topical application \\ Interaction with the stratum corneum}

The SC is made of protein-rich cells embedded in a lipid extracellular matrix. This arrangement has been referred to as the bricks and mortar model..$^{28}$ In addition, corneocytes are anchored together by means of intercellular proteic structures called corneodesmosomes. ${ }^{29}$ Depending on their composition, size, and shape, macromolecules and supramolecular carriers interact differently with this very complex barrier. It has been postulated that lipid-based particles have the tendency to merge with the skin lipid layers, whereas polymeric particles, being more hydrophilic, might have different interactions with the extracellular lipids of the SC. ${ }^{30}$ However, even if the components of polymeric carriers do not fuse with the lipids of the SC, the interaction with the SC may change their threedimensional conformation leading to adsorption, destabilization, or agglomeration. Furthermore, the SC is covered with sebum, rich in lipids and proteins, which can interact with the applied polymeric materials destabilizing them or changing the way they interact with other skin components. A number of different commensal bacteria strains reside on the skin surface, in skin furrows and appendages and might interact with applied materials. The $\mathrm{pH}$ on the surface of the $\mathrm{SC}$ is $4.2-5.6 .{ }^{31}$ Such an acidic $\mathrm{pH}$ can influence the colloidal stability of particles. The acidic $\mathrm{pH}$ increases the rate of hydrolysis and the activity of $\mathrm{pH}$-sensitive enzymes, which in turn might accelerate the degradation of polymeric particles. ${ }^{32}$ Finally, the SC is a dynamically evolving structure and, near to a more compact region (stratum compactum), a looser part can be distinguished due to desquamation processes (stratum disjunctum; Figure 1). Thus, substances accumulated in the stratum disjunctum are rapidly eliminated by the shedding of the outermost layers, whereas the SC can function as a reservoir for those particulate materials penetrating deeper. Once released, the drugs can diffuse across the $\mathrm{SC}$ to the viable skin layers. Three
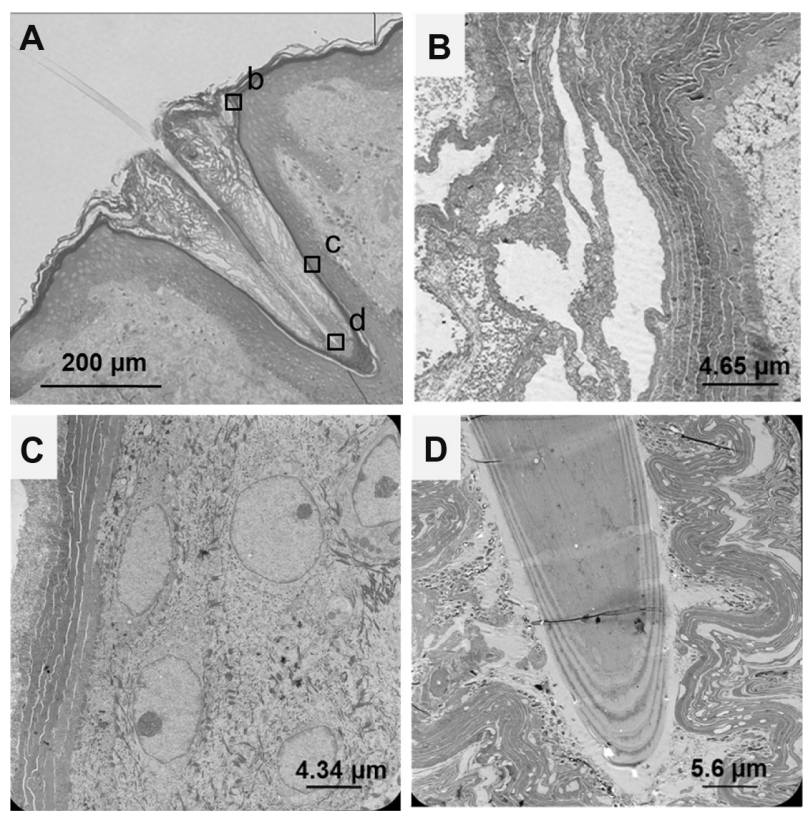

Figure I Human terminal hair follicle. (A) Light microscopy image of a longitudinal section showing the hair follicle infundibulum and the hair shaft. The infundibulum is filled with cell debris and sebum. Insets $(b-d)$ are regions of the hair follicle that were observed by means of transmission electron microscopy ( $B, C$ and $D$ respectively), using an ultrafine section prepared from the same sample block. (B) The SC in the apical part of the hair follicle orifice consists of several corneocytes layers. Bacteria colonies are visible as well. (C) The SC in the hair follicle infundibulum is thinner than the SC in the upper part of the hair follicle orifice and in the inter-follicular epidermis. (D) Image of the hair shaft and the inner root sheath.

Abbreviation: SC, stratum corneum.

penetration pathways have been described for topically applied substances: the intercellular, the transcellular, and the transfollicular route. ${ }^{33-35}$ In general, the penetration pathway and rate of diffusion of released drugs depend on their lipophilicity/ hydrophilicity, their molecular weight, and the carrier type.

\section{Hair follicle pathway}

The hair follicle canal of the pilosebaceous unit represents a reservoir where micro-, submicron-, and nano-sized material can penetrate and accumulate (Figure 1).

It took a while before the hair follicle was taken into consideration as a possible portal to skin penetration. In fact, hair follicle orifices represent only $0.1 \%$ of the skin surface $^{36}$ and it was assumed that the sebum impedes the penetration of material in the hair follicle canal. Only in the last decade a number of studies demonstrated the importance of the transfollicular pathway, especially for the preferential penetration of particulate materials. Particles that accumulate in the hair follicle are protected from SC turnover and persist for days before they are cleared. ${ }^{37}$ The size of the hair follicle orifices ranges between $172 \pm 70 \mu \mathrm{m}$ in terminal hair follicles and $86 \pm 37 \mu \mathrm{m}$ in vellus hair follicles, ${ }^{38}$ and the interaction surface of hair follicle infundibulum was calculated to be 
approximately 0.68 and $0.068 \mathrm{~cm}^{2}$ for empty or full follicles, respectively. ${ }^{39}$ Consequently, the hair follicle infundibulum represents not only a reservoir but also a considerable surface area where interaction between accumulated material and skin structures can occur. The hair follicle is a shunt for a more rapid penetration of substances. When the hair follicle orifices were selectively closed, the applied substances reached the same blood values in volunteers with open hair follicles. ${ }^{40,41}$ This might be due to the fact that the SC barrier in the deep infundibulum is thinner (Figure 1C) and molecules can diffuse more rapidly here than on the skin surface where the $\mathrm{SC}$ consists of several more layers (Figure 1B). In addition, those carriers accumulated in the hair follicle canal release the loaded drug, thereby generating a high local concentration of the drug which is a driving force for its diffusion to the viable skin. The removal of the hair follicle content (sebum, cells debris, and eventually bacteria) favors the penetration of topically applied particulate materials. ${ }^{42}$

\section{Translocation across}

\section{the stratum corneum}

Even if the debate on the translocation of particulate material across the SC is not yet closed, in general, it is believed that only lipophilic molecules with a molecular weight below 500 Dalton can pass the skin barrier, whereas the penetration of hydrophilic molecules, macromolecules, and nanoparticles is significantly impaired. ${ }^{43-45}$ Liposomes, solid lipid nanoparticles, and polymeric DDS were shown to enhance the skin penetration of loaded substances but there is no evidence for the translocation of entire particles across the intact SC of healthy skin. On the contrary, it seems that components of the lipid-based particles dissolve into the lipids of the SC enhancing its permeability. ${ }^{46,47}$ In the case of polymer-based carriers, data on the penetration across the SC are poor due to the fact that most of the studies cited in this article detected the released fluorochrome (or drug), but only a few studies attempted to find out the fate of the carrier after topical application. Interestingly, there is one report claiming that 200-250 nm poly( $N$-isopropylacrylamide) (NIPAM) and poly(N-isopropylacrylamide-co-acrylic acid) (NIPAM-co-AAc) nanogels penetrated across epidermal sheets of pig ears. Indeed, these biocompatible carriers were detectable by means of transmission electron microscopy (TEM) in the acceptor medium of the Franz diffusion cell. ${ }^{48}$ However, these results apply to epidermal sheets isolated by heating at $60^{\circ} \mathrm{C}$ (which might damage the lipid matrix and corneodesmosomes) and not to full thickness skin. This underlines how challenging it is to visualize and elucidate the interaction between carriers and the skin barrier at the subcellular level.

\section{Carriers penetration across disrupted skin barriers}

When developing DDS for skin disorders, the different features of diseased skin have to be considered. Whereas in healthy skin particulate carriers penetrate in the SC and in the hair follicle canals releasing there the loaded drugs (Figure 2A), in unhealthy skin, where the integrity of the $\mathrm{SC}$ is altered, carrier systems might cross the SC barrier and enter the epidermis and dermis (Figure 2B). Several methods have been developed to bypass the $\mathrm{SC}$, for example by disrupting it with adhesive tapes and abrasion, ${ }^{49}$ altering it with permeabilizing agents, ${ }^{50,51}$ or using microneedles. ${ }^{52}$ Most of these procedures were tested for transdermal drug delivery or transcutaneous vaccination strategies. Only a small number of studies investigated the penetration of particles across diseased animal or human skin. ${ }^{53,54}$ In fact, most animal studies report the diffusion of the released drugs and their efficacy but not on the fate of the used carrier systems. ${ }^{54-56}$ Most authors found significantly higher drug permeability in inflamed skin than in healthy skin. ${ }^{55,56}$ These results are explained with the swelling of the tissue and the increased intercellular fluid in the epidermis which might alter the structure of tight junctions. However no direct evidence for nanoparticle translocation was provided.

In general, those studies using ultraviolet ${ }^{57}$ irradiation or mechanical methods to disrupt the SC and activate the immune system showed that topically applied nano-materials can be detected in the viable skin layers. ${ }^{58-60}$ Once particles cross the SC, they might also reach the subcutaneous lymph nodes after being taken up by skin antigen presenting cells or by passive transport following the lymph drainage. ${ }^{61}$ All these findings underline the need for further knowledge on the interactions and fate of the carrier system after topical application on diseased skin.

Most skin diseases are associated with different degrees of SC disruption. ${ }^{29}$ Disease-specific differences, however, can have a tremendous impact on how carrier systems interact with skin. In atopic dermatitis and contact eczema, defects in expression of tight junction components and filaggrin are responsible for the dysfunction of the SC resulting in a higher susceptibility to external allergens and opportunistic infections. ${ }^{62}$ Thus, it is reasonable to think that particulate materials have easy access. Nanoparticles which can penetrate the epidermis and dermis can interact with immune system cells and be taken up by phagocytic cells. 


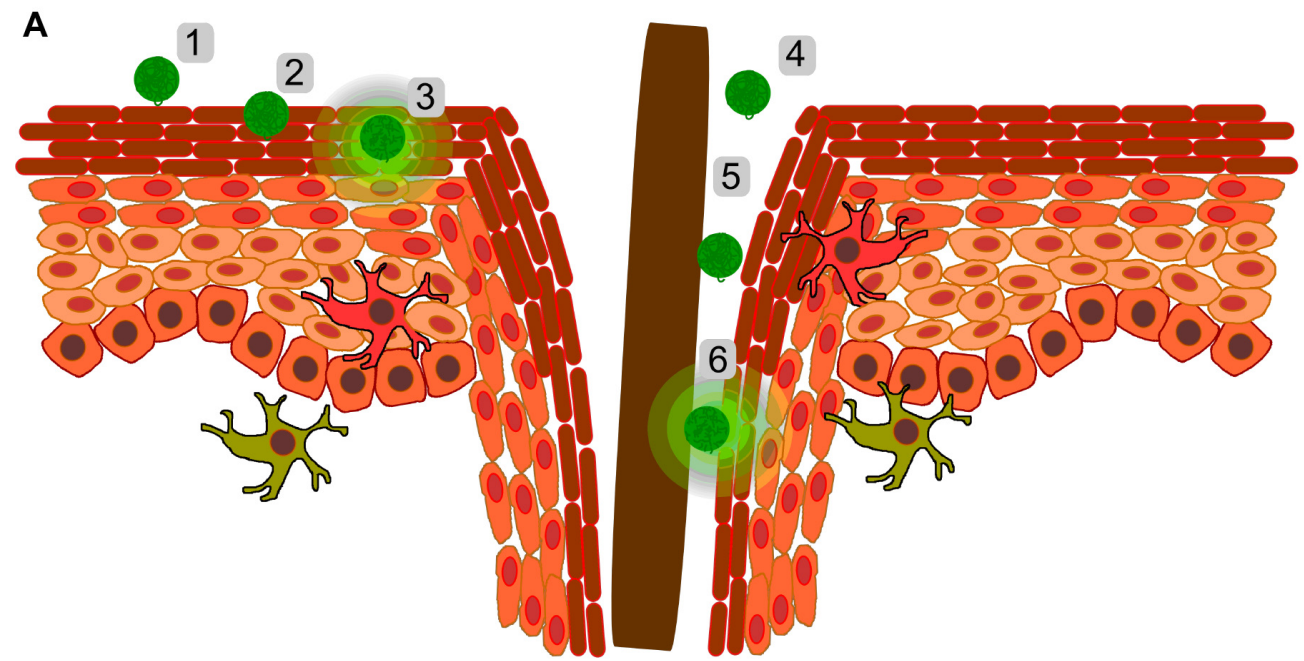

B

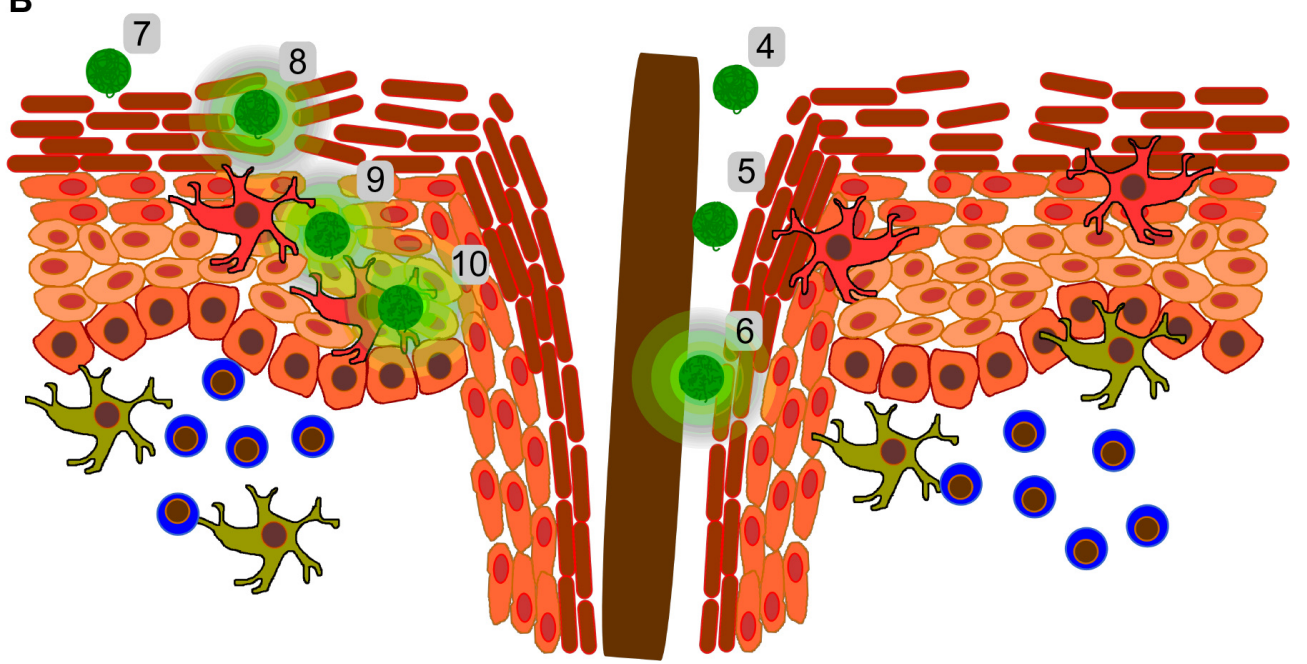

Figure 2 Skin penetration of polymeric carriers and drug release in (A) healthy skin and (B) hypothesis of polymer particle behavior in diseased skin. Notes: (A) When the skin barrier is intact, topically applied carriers interact with the skin surface $(I, 4)$ and accumulate in the SC (2) and in the hair follicle canal $(5)$. There, they degrade and/or release the loaded substances $(3,6)$. (B) In most skin conditions, the skin barrier is disrupted and the skin immune system is activated. Topically applied substances interact with the altered skin surface (7), penetrate across the SC (8), enter the skin viable layers (9), and can be taken up by activated dendritic cells or macrophages (10). Polymeric particles can degrade and/or deliver their loads in the SC (8), in the intercellular space (9), or upon internalization in the cells (I0).

Abbreviation: SC, stratum corneum.

Because atopic and contact dermatitis are associated with altered immune responses, certain nanoparticles might exacerbate the symptoms of the pathology, as was found for polystyrene nanoparticles. ${ }^{63}$ In psoriasis, keratinocyte hyperproliferation and abnormal SC stacking leads to epidermal thickening and the formation of scales. The cytokine milieu induces vasodilatation and cell infiltration. Even if the SC is thicker, tight junction proteins are altered leading to increased skin permeability to topically applied drugs. ${ }^{64}$ Nevertheless, this does not apply to all types of molecules and nanoparticles. In fact, Prow et al found that penetration of $\mathrm{ZnO}_{2}$ nanoparticles was not increased in psoriatic skin compared to a non-lesional skin region of the same patient. ${ }^{65}$ However, no data are available with regard to the penetration of more flexible particles like polymer-based nanocarriers. Skin barrier properties are also altered during skin infection. For example, dermatophytes secrete proteolytic enzymes which degrade the SC allowing them to invade and disseminate through $\mathrm{it}^{66}$ and at the same time altering skin permeability. In general, during bacterial and fungal infections, the SC is more permeable and the skin immune system is activated with the presence of cell infiltrates and vasodilatation similar to the situation found in eczema. Nevertheless, depending on the type of pathogen and the stage of disease, different degrees of inflammation, skin dryness, and desquamation are present. The skin barrier is compromised in aged skin as well. ${ }^{67} \mathrm{It}$ has been shown that elderly skin is dry and scaly. The reason for these defects is related to lower lipid levels in lamellar 
bodies ${ }^{68}$ and a decrease in epidermal filaggrin. ${ }^{69}$ In addition, aged skin manifests epidermal and dermal atrophy, flattening of the epidermal rete ridges, as well as reduced numbers of fibroblasts and mast cells. ${ }^{67}$

On the other hand, there are other skin conditions that are characterized by a less permeable skin barrier. In hypertrophic scars, for example, even if the amount of hydrophobic ceramide is decreased, the SC is irregular and thicker. Another example is acne vulgaris, which is characterized by a hyperproliferation of keratinocytes in the hair follicle at the level of the sebaceous gland duct and an increased production of sebum which result in a strong barrier to the penetration of hydrophilic molecules and nanoparticles.

\section{Cellular uptake}

Once a carrier system or a drug has successfully crossed the skin barrier and has reached the viable epidermis layers, it can diffuse across the extracellular space and/or be taken up by epidermis and dermis cells (Figure 2B). Whereas small amphiphilic drugs can diffuse across the cell membrane, macromolecules like proteins, nucleic acids, as well as nanoand submicron-material can be internalized by cells only via the energy-dependent mechanism of endocytosis. ${ }^{70,71}$ In general, only certain types of cells can internalize particulate material with size above $200 \mathrm{~nm} .{ }^{70}$ For example, when dendritic cells were co-cultured with T-cells and incubated with fluorescently labeled bacteria and polystyrene particles (200 nm), we found that only dendritic cells internalized both particles (unpublished data, Figure 3).

Langerhans cells in the epidermis are cells specialized in taking up particulate materials penetrating across a disrupted skin barrier. Also, keratinocytes, which are the most represented cells in the epidermis $(90 \%)$, can internalize
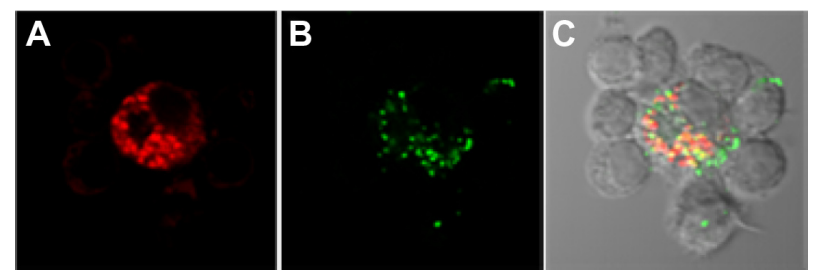

Figure 3 Confocal fluorescence images showing the uptake of Escherichia coli particles and polystyrene particles $(200 \mathrm{~nm})$ by dendritic cells (unpublished data). Dendritic cells derived from peripheral blood mononuclear cells were co-cultured with T-cells and incubated with inactivated $E$. coli particles labeled with a $\mathrm{pH}$ sensitive dye that becomes fluorescent in an acidic environment (A; red) and FITC-loaded $200 \mathrm{~nm}$ polystyrene particles (B, green). The overlay image (C) shows that the bigger cells (ie, dendritic cells), but not T-cells, had internalized particles. Both $E$. coli and polystyrene particles were co-localized in intracellular acidic compartments.

Abbreviation: FITC, fluorescein isothiocyanate. penetrated materials. Nevertheless, in vitro experiments with freshly isolated epidermis cells have shown that, depending on the particle size, keratinocytes are less prone than Langerhans cells to take up particles. ${ }^{59}$ In the dermis, penetrated material comes in contact with cells like fibroblasts, mast cells, and T-cells. However, macrophages and dendritic cells are most likely to internalize them. Once internalized, the carrier systems can undergo degradation in the acid and hydrolytic environment of lysosomes and release the loaded drug. ${ }^{72}$ Hydrolysis and enzymatic degradation may be exploited as a mechanism for drug release, though the same reactions may be deleterious for certain loaded molecules such as proteins or nucleic acids. ${ }^{23}$ To avoid degradation, endosomal escape has to be achieved. ${ }^{73}$ On the other hand, for vaccination purposes, the uptake of antigen-loaded carriers by antigen presenting cells and their intracellular degradation is advantageous, being the steps necessary for major histocompatibility complex (MHC)-I and MHC-II presentation. ${ }^{22}$

\section{Polymer degradation and drug release}

Once applied on the skin surface, polymeric biomaterials may be degraded by chemical and/or enzymatic reactions depending on the nature of the polymer. Hydrolytic enzymes, including proteases, esterases, glycosidases, and phosphatases, can catalyze the water mediated cleavage of different chemical bonds. The degradation of polymer particles is often a complex process and the release of the loaded drug exhibits often non-linear kinetics. The degradation of the polymer may interfere with the release of the drug, inhibiting or accelerating it, or might degrade the loaded drugs. ${ }^{74}$ Ideally, a biodegradable polymer should decompose into non-toxic products and should degrade in a controlled manner. In this way, not only the safety but also the selectivity and efficacy of the drug are enhanced. For example, hydrolysis of the drug-carrier bond can be controlled by introducing linkers cleavable by enzymes that are localized predominantly in the diseased tissue or target cell. ${ }^{75}$ These processes are influenced by skin parameters like temperature, $\mathrm{pH}$, and protein concentration. ${ }^{76,77}$ Also, it is possible to include degrading enzymes in the polymer matrix. ${ }^{74}$ The type of polymer, the nature of interactions between drug and polymer, and the affinity of the drug to skin components determine the time and the place where the carrier is degraded and the drug released. Polymers can be readily hydrolyzed after penetration in the $\mathrm{SC}$, upon penetration in the epidermis and dermis, or only after cellular uptake (Figure 2B). 
The release of a loaded drug may also take place by other processes like conformational changes of the particles (destabilization, swelling, or shrinkage). Upon topical application, drugs adsorbed on the carrier surface may be released by affinity-driven diffusion or by exchange with skin components like proteins. The physicochemical properties of the carriers seem to play an important role in drug release and diffusion. ${ }^{78,79}$

\section{Challenges for carrier-based dermatotherapies}

Whereas transdermal delivery implies the transport of actives across the whole healthy skin to reach the systemic compartment or regions beneath the skin (eg, skeletal or muscular tissues), the treatment of skin pathological conditions implies the local delivery of actives to skin regions with altered parameters. The knowledge on the pathophysiology and mechanisms underlying a number of skin conditions has enormously increased in the past years, showing that diseased skin is morphologically, physiologically, and biologically different from healthy skin. ${ }^{80}$ Thus, different strategies can be developed taking into consideration the special conditions which characterize affected skin and exploiting them to achieve a more selective delivery of the drug.

\section{Skin inflammatory conditions}

The treatment of atopic dermatitis, contact eczema, and psoriasis is achieved using anti-inflammatory drugs (steroidal and non-steroidal) or immunomodulatory and immunosuppressive drugs (eg, tacrolimus). These actives are administered topically or, in severe cases, orally or intravenously. One of the main problems of topical treatment with glucocorticoids is to reach effective concentrations of the anti-inflammatory drug in the epidermis avoiding drug localization in the dermis or its rapid diffusion to the systemic compartment. Glucocorticoid localization in the dermis leads to induction of metalloproteases and degradation of connective tissue, resulting in thinning of skin and skin atrophy. On the other hand, the rapid clearance of the drug from skin to the systemic compartment is responsible for reduction of drug efficacy. Nanocarriers offer the possibility to improve the efficacy and safety of corticosteroids by creating depots in the SC, hair follicle canal, and epidermis as well as local release of the drug in a sustained manner. ${ }^{81,82}$ In the case of macrolide drugs, like tacrolimus, there is a need for a better penetration across the SC especially in the case of psoriasis where keratinocytes over-proliferation results in plaque formation.
In fact, the high molecular weight and the hydrophilic nature of the molecule impede its diffusion into the skin. The use of carrier systems that can increase its penetration across the $\mathrm{SC}$ and thereby protect it from degradation would increase the efficacy of this topical treatment. Furthermore, a better and more selective delivery of the drug to the dendritic and T-cell infiltrates in inflamed skin would reduce the side effects and increase the therapeutic activity of the drug. It is now recognized that skin dendritic cell subsets are involved in chronic inflammatory skin conditions and in the initiation of skin allergic reaction. Therefore, particle-based targeting of dendritic cells and their modulation seems to be a promising strategy against such skin conditions where an overreaction of skin immune cells is involved. ${ }^{83}$

The skin penetration profile of small hydrophobic molecules, like non-steroidal anti-inflammatory drugs, can also be improved by encapsulation in particles. The delivery of flufenamic acid by PLGA particles resulted in a more prolonged and localized drug release in the epidermis, whereas surface modified PLGA and chitosan nanoparticles formulated in a gelling agent were successfully used to deliver spantide II and ketoprofen to allergic contact dermatitis and psoriatic plaque-like skin models. ${ }^{24,54}$ Polymer particles, because of their ability to protect encapsulated drugs, also have the potential to optimize the topical delivery of nucleic acid molecules, such as small interfering ribonucleic acid (siRNA) for the regulation of cytokines' expression and control of inflammatory skin diseases. ${ }^{84}$

\section{Skin infections}

The therapy of skin infections can also profit from a more selective and effective delivery of the antimicrobial drug. Especially in the case of pathogens colonizing the lower infundibulum, the use of nanocarriers that accumulate in the hair follicle canal and thus the achievement of high concentrations of antimicrobials can decisively improve the outcome of a topical therapy. Another challenge is the reduction of unwanted toxicity of antimicrobials toward skin cells. In the case of antibiotic-resistant strains, besides the development of new generations of antibiotics, new therapeutic approaches include the application of DDS for the slow but sustained release of toxic substances such as metal ions or nitric oxide, thus limiting their side effects. For example, hydrogel (polyethylene glycol, chitosan)-glass composites have been tested for the sustained release of nitric oxide, whereas silver nanoparticles are used as a source of silver ions. ${ }^{85,86}$ Biodegradable gelatin fibers loaded with antimicrobial silver nanoparticles 
were also tested as dressing materials in the treatment of wounds and chronic wounds. ${ }^{87}$

\section{Hair follicle associated disorders}

The targeting of drugs to the pilosebaceous unit by means of nano- and submicron carriers can be exploited to treat hair follicle-associated inflammation, hair growth, and sebaceous gland disorders like alopecia and acne, respectively. For example, to treat alopecia androgenetica the drug should be targeted to the hair bulb where the hair shaft is generated. For this purpose, minoxidil was formulated on $40 \mathrm{~nm}$ poly( $\varepsilon$-caprolactone)-block-poly(ethyleneglycol) carriers with positive results. ${ }^{88}$ For the treatment of acne vulgaris, the drug should be targeted to the infundibulum at the level of the sebaceous gland. Thus, microspheres accumulating preferentially in the hair follicle infundibulum would be the carrier of choice. ${ }^{89}$ The release of the loaded drug can take place due to diffusion gradients or can be triggered by the special environment within the hair follicle canal. ${ }^{90}$ We have shown that $228 \mathrm{~nm}$ PLA particles accumulated in the hair follicle infundibulum realizing a sustained release of the loaded lipophilic dye nile red for up to 24 hours..$^{91}$ Later, we found that the type of loaded dye also played an important role with regard to release kinetics and stability of the PLA particles in the hydrophobic environment of the hair follicle infundibulum. ${ }^{90}$ Carrier systems can also improve the tolerability of retinoids for the treatment of acne. Despite their promising anti-aging and anti-comedolitic properties, these drugs can cause irritation and pain and their encapsulation in carrier systems can help to reduce this side effect. Most studies used lipid-based carriers but polymers have also been used in combination with emulsions or lipid carrier systems. For example, Cho et al used PEG-PCL-PEO triblock copolymers to stabilize retinol emulsions, whereas Ridolfi et al used chitosan-solid lipid nanoparticles to delivery tretinoin. ${ }^{92,93}$

The targeting of drugs to perifollicular immune system cells by means of polymer particles might be useful for the treatment of alopecia areata. This autoimmune disease is characterized by infiltrating cluster of differentiation (CD) 4 and CD8 T-cells directed against auto-antigens and results in the destruction of the hair shaft. The targeted inhibition of overreacting cells by means of particulate carriers might be a possible therapy. For example, $75 \mu \mathrm{m}$ cationized gelatine particles were used to deliver siRNA as a new therapeutic approach, targeting the T-box 21 gene (Tbx21) to repress the expression of interferon- $\gamma$ gene. ${ }^{94}$ Another possible treatment is the topical application of diphenylcyclopropenone (DCP), which is an irritant and diverts T-cells from the hair shaft. However, the treatment is painful (irritation) and can be done only by trained personnel. The use of particles might allow a slow but sustained release of the substance minimizing the irritating effect.

\section{Skin cancer}

Polymeric carrier systems like dendrimers, microspheres, and nanogels, besides lipid-based carriers, are ideal systems for the topical delivery of chemotherapeutic agents and siRNAs to treat skin malignancies. ${ }^{95}$ In fact, the topical delivery of anti-proliferative agents and photosensitizing agents has the advantage of reducing side effects and improving the compliance of the patients. Such carrier systems, eg, PLA or PAMAM based particles, have been tested for the topical delivery of drugs to different types of skin tumors. ${ }^{96-99}$

\section{Targeting the skin immune system}

Skin possesses a large number of immune active cells, from keratinocytes to different sub-types of skin resident T-cells, macrophages, dendritic cells, and mast cells. The fact that mainly dendritic cells are prone to take up particulate material suggested using submicron carriers to target this cell population. ${ }^{100}$ Dendritic cells are potent antigen presenting cells and represent the bridge between innate and adaptive immune response. It has been shown that targeting antigens to the skin by means of virus particles (MVA), but also using PLA particles, induces potent cellular responses as well as skin and mucosal humoral immunity. ${ }^{101,102}$ Thus, different types of nanoparticles are actually under investigation as vaccine delivery systems against cancer and infectious diseases. ${ }^{103}$ In order to deliver a sufficient amount of antigen to skin dendritic cells, it is important that particles are delivered across the skin barrier and that they are taken up by the target cells. For this reason, different physical methods (eg, stripping, microneedles, electroporation, or iontophoresis) have been developed to overcome the skin barrier. ${ }^{104}$ Thus, for vaccination purposes it is mandatory to use biodegradable and compatible material to avoid intolerance responses or accumulation of particles in secondary lymphatic organs.

\section{Gene therapy}

Another special challenge for drug delivery is the targeting of therapeutic genes to skin cells. Very promising is also the delivery of siRNA molecules in order to control the post-transcriptional pathways of gene expression. To achieve this goal several obstacles have to be overcome. The deoxyribonucleic acid (DNA) or RNA moieties has to be transported across the skin barrier, be protected from degrading nucleases, be taken up by cells, and reach its target 
in the cytoplasm or in the cell nucleus. Polymer materials are intensively investigated as delivery agents because of the possibility to protect the nucleic acid and to achieve a sustained release and expression of the therapeutic gene. Despite all obstacles, there are encouraging reports describing the successful in vivo expression of genes after topical delivery of DNA loaded on cationic or PEGylated liposome as well as chitosan nanoparticles. ${ }^{105-107}$ Tissue growth, vaccination, anticancer treatment, and anti-inflammatory therapy can be achieved by gene transfer or regulation of gene expression. ${ }^{108,109}$ Principally, all skin diseases with a genetic basis could be treated by delivery of the missing or dysfunctional gene.

\section{Conclusion}

Several studies showed the efficacy and advantages of biodegradable polymeric materials as carriers or delivery systems in dermatology. These materials are safe and offer tools to develop carrier systems tailored for each specific dermatological disease. For this purpose, future work should focus on a more detailed knowledge of the interactions occurring between polymeric carrier systems and skin environment. Drug permeation and efficacy measurements should be complemented by detailed investigation on carrier skin penetration, uptake by skin cells, mechanism of degradation and drug-release, as well as toxicological properties. Also, it is important that such investigations are conducted using skin models of the specific pathological conditions to be treated. Investigating the interaction of delivery systems with disrupted skin barrier or activated immune system will allow tailoring polymeric materials in order to enhance the selectivity and specificity of drug delivery. The fact that new polymer-based carrier systems are being developed and that the number of their dermatological applications is constantly increasing demonstrates the huge potential of these biodegradable and versatile materials.

\section{Acknowledgments}

We thank P Schrade and Prof Dr S Bachmann (Department of Electron Microscopy, Charité - Universitätsmedizin Berlin, Germany) for the electron microscopy imaging of the human hair follicle. This study was supported by the Deutsche Forschung Gesellschaft, SFB1112 - Nanocarrier: Architektur, Transport und zielgerichtete Applikation von Wirkstoffen für therapeutische Anwendungen.

\section{Disclosure}

The authors report no conflicts of interest in this work.

\section{References}

1. Papakostas D, Rancan F, Sterry W, Blume-Peytavi U, Vogt A. Nanoparticles in dermatology. Arch Dermatol Res. 2011;303(8): 533-550.

2. Egbaria K, Weiner N. Liposomes as a topical drug delivery system. Adv Drug Deliver Rev. 1990;5(3):287-300.

3. Müller RH, Radtke M, Wissing SA. Solid lipid nanoparticles (SLN) and nanostructured lipid carriers (NLC) in cosmetic and dermatological preparations. Adv Drug Deliv Rev. 2002;54 Suppl 1:S131-S155.

4. Guterres SS, Alves MP, Pohlmann AR. Polymeric nanoparticles, nanospheres and nanocapsules, for cutaneous applications. Drug Target Insights. 2007;2:147-157.

5. Zhong SP, Zhang YZ, Lim CT. Tissue scaffolds for skin wound healing and dermal reconstruction. Wiley Interdiscip Rev Nanomed Nanobiotechnol. 2010;2(5):510-525.

6. Venugopal J, Ramakrishna S. Biocompatible nanofiber matrices for the engineering of a dermal substitute for skin regeneration. Tissue Eng. 2005;11(5-6):847-854.

7. Ekaputra AK, Prestwich GD, Cool SM, Hutmacher DW. The threedimensional vascularization of growth factor-releasing hybrid scaffold of poly (epsilon-caprolactone)/collagen fibers and hyaluronic acid hydrogel. Biomaterials. 2011;32(32):8108-8117.

8. Hadjizadeh A, Doillon CJ. Directional migration of endothelial cells towards angiogenesis using polymer fibres in a $3 \mathrm{D}$ co-culture system. J Tissue Eng Regen Med. 2010;4(7):524-531.

9. Wichterle O, Lim D. Hydrophilic gels for biological use. Nature. 1960;185:117-118.

10. Hoffman AS. Hydrogels for biomedical applications. Adv Drug Deliv Rev. 2002;54(1):3-12.

11. Arts JH, Muijser H, Duistermaat E, Junker K, Kuper CF. Five-day inhalation toxicity study of three types of synthetic amorphous silicas in Wistar rats and post-exposure evaluations for up to 3 months. Food Chem Toxicol. 2007;45(10):1856-1867.

12. Bettencourt A, Almeida AJ. Poly(methyl methacrylate) particulate carriers in drug delivery. J Microencapsul. 2012;29(4):353-367.

13. Rehman K, Zulfakar MH. Recent advances in gel technologies for topical and transdermal drug delivery. Drug Dev Ind Pharm. 2013.

14. Chen X, Peng LH, Shan YH, et al. Astragaloside IV-loaded nanoparticleenriched hydrogel induces wound healing and anti-scar activity through topical delivery. Int J Pharm. 2013;447(1-2):171-181.

15. Moya-Ortega MD, Alvarez-Lorenzo C, Concheiro A, Loftsson T. Cyclodextrin-based nanogels for pharmaceutical and biomedical applications. Int J Pharm. 2012;428(1-2):152-163.

16. Asadian-Birjand M, Sousa-Herves A, Steinhilber D, Cuggino JC, Calderon M. Functional nanogels for biomedical applications. Curr Med Chem. 2012;19(29):5029-5043.

17. Sasaki Y, Akiyoshi K. Nanogel engineering for new nanobiomaterials: from chaperoning engineering to biomedical applications. Chem Rec. 2010;10(6):366-376.

18. Dang JM, Leong KW. Natural polymers for gene delivery and tissue engineering. Adv Drug Deliv Rev. 2006;58(4):487-499.

19. Elzoghby AO, Samy WM, Elgindy NA. Protein-based nanocarriers as promising drug and gene delivery systems. J Control Release. 2012; 161(1):38-49.

20. Cho K, Wang X, Nie S, Chen ZG, Shin DM. Therapeutic nanoparticles for drug delivery in cancer. Clin Cancer Res. 2008;14(5):1310-1316.

21. Feng Y, Lu J, Behl M, Lendlein A. Progress in depsipeptide-based biomaterials. Macromol Biosci. 2010;10(9):1008-1021.

22. Fahmy TM, Demento SL, Caplan MJ, Mellman I, Saltzman WM. Design opportunities for actively targeted nanoparticle vaccines. Nanomedicine (Lond). 2008;3(3):343-355.

23. Panyam J, Labhasetwar V. Biodegradable nanoparticles for drug and gene delivery to cells and tissue. Adv Drug Deliv Rev. 2003;55(3): 329-347.

24. Luengo J, Weiss B, Schneider M, et al. Influence of nanoencapsulation on human skin transport of flufenamic acid. Skin Pharmacol Physiol. 2006;19(4):190-197. 
25. Brannon-Peppas L. Recent advances on the use of biodegradable microparticles and nanoparticles in controlled drug delivery. Int J Pharm. 1995;116(1):1-9.

26. Rösler A, Vandermeulen GW, Klok HA. Advanced drug delivery devices via self-assembly of amphiphilic block copolymers. Adv Drug Deliv Rev. 2001;53(1):95-108.

27. Stiriba SE, Frey H, Haag R. Dendritic polymers in biomedical applications: from potential to clinical use in diagnostics and therapy. Angew Chem Int Ed Engl. 2002;41(8):1329-1334.

28. Michaels AS, Chandrasekaran SK, Shaw JE. Drug permeation through human skin: theory and in vitro experimental measurement. AICHEJ. 1975;21(5):985-996.

29. Proksch E, Brandner JM, Jensen JM. The skin: an indispensable barrier. Exp Dermatol. 2008;17(12):1063-1072.

30. Bouwstra JA, Honeywell-Nguyen PL. Skin structure and mode of action of vesicles. Adv Drug Deliv Rev. 2002;54 Suppl 1:S41-S55.

31. Schmid-Wendtner MH, Korting HC. The $\mathrm{pH}$ of the skin surface and its impact on the barrier function. Skin Pharmacol Physiol. 2006;19(6): 296-302.

32. Rawlings AV, Voegeli R. Stratum corneum proteases and dry skin conditions. Cell Tissue Res. 2013;351(2):217-235.

33. Simonetti O, Hoogstraate AJ, Bialik W, et al. Visualization of diffusion pathways across the stratum corneum of native and in-vitroreconstructed epidermis by confocal laser scanning microscopy. Arch Dermatol Res. 1995;287(5):465-473.

34. Wang TF, Kasting GB, Nitsche JM. A multiphase microscopic diffusion model for stratum corneum permeability. II. Estimation of physicochemical parameters, and application to a large permeability database. J Pharm Sci. 2007;96(11):3024-3051.

35. Lademann J, Knorr F, Richter H, et al. Hair follicles - an efficient storage and penetration pathway for topically applied substances. Summary of recent results obtained at the Center of Experimental and Applied Cutaneous Physiology, Charité-Universitätsmedizin Berlin, Germany. Skin Pharmacol Physiol. 2008;21(3):150-155.

36. Szabo G. The number of eccrine sweat glands in human skin. Adv Biol Skin. 1962;3:1-5.

37. Lademann J, Richter H, Teichmann A, et al. Nanoparticles - an efficient carrier for drug delivery into the hair follicles. Eur J Pharm Biopharm. 2007;66(2):159-164.

38. Vogt A, Hadam S, Heiderhoff M, et al. Morphometry of human terminal and vellus hair follicles. Exp Dermatol. 2007;16(11):946-950.

39. Schwartz JR, Shah R, Krigbaum H, Sacha J, Vogt A, Blume-Peytavi U. New insights on dandruff/seborrhoeic dermatitis: the role of the scalp follicular infundibulum in effective treatment strategies. Br J Dermatol. 2011;165 Suppl 2:18-23.

40. Blume-Peytavi U, Massoudy L, Patzelt A, et al. Follicular and percutaneous penetration pathways of topically applied minoxidil foam. Eur J Pharm Biopharm. 2010;76(3):450-453.

41. Trauer S, Lademann J, Knorr F, et al. Development of an in vitro modified skin absorption test for the investigation of the follicular penetration pathway of caffeine. Skin Pharmacol Physiol. 2010;23(6):320-327.

42. Toll R, Jacobi U, Richter H, Lademann J, Schaefer H, Blume-Peytavi U. Penetration profile of microspheres in follicular targeting of terminal hair follicles. J Invest Dermatol. 2004;123(1):168-176.

43. Watkinson AC, Bunge AL, Hadgraft J, Lane ME. Nanoparticles do not penetrate human skin - a theoretical perspective. Pharm Res. 2013;30(8):1943-1946.

44. Baroli B. Penetration of nanoparticles and nanomaterials in the skin: fiction or reality? J Pharm Sci. 2010;99(1):21-50.

45. Nohynek GJ, Lademann J, Ribaud C, Roberts MS. Grey goo on the skin? Nanotechnology, cosmetic and sunscreen safety. Crit Rev Toxicol. 2007;37(3):251-277.

46. Schreier H, Bouwstra J. Liposomes and niosomes as topical drug carriers: dermal and transdermal drug delivery. J Control Rel. 1994;30(1):1-15.

47. Fang JY, Hong CT, Chiu WT, Wang YY. Effect of liposomes and niosomes on skin permeation of enoxacin. Int J Pharm. 2001;219(1-2): $61-72$.
48. Samah NA, Williams N, Heard CM. Nanogel particulates located within diffusion cell receptor phases following topical application demonstrates uptake into and migration across skin. Int J Pharm. 2010; 401(1-2):72-78.

49. Zhang LW, Monteiro-Riviere NA. Assessment of quantum dot penetration into intact, tape-stripped, abraded and flexed rat skin. Skin Pharmacol Physiol. 2008;21(3):166-180.

50. Prow TW, Monteiro-Riviere NA, Inman AO, et al. Quantum dot penetration into viable human skin. Nanotoxicology. 2012;6(2): 173-185.

51. Lane ME. Skin penetration enhancers. Int J Pharm. 2013;447(1-2): 12-21.

52. Prausnitz MR. Microneedles for transdermal drug delivery. Adv Drug Deliv Rev. 2004;56(5):581-587.

53. Lin LL, Grice JE, Butler MK, et al. Time-correlated single photon counting for simultaneous monitoring of zinc oxide nanoparticles and $\mathrm{NAD}(\mathrm{P}) \mathrm{H}$ in intact and barrier-disrupted volunteer skin. Pharm Res. 2011;28(11):2920-2930.

54. Shah PP, Desai PR, Patel AR, Singh MS. Skin permeating nanogel for the cutaneous co-delivery of two anti-inflammatory drugs. Biomaterials. 2012;33(5):1607-1617.

55. Kang MJ, Eum JY, Jeong MS, et al. Facilitated skin permeation of oregonin by elastic liposomal formulations and suppression of atopic dermatitis in NC/Nga mice. Biol Pharm Bull. 2010;33(1):100-106.

56. Abdel-Mottaleb MM, Moulari B, Beduneau A, Pellequer Y, Lamprecht A. Nanoparticles enhance therapeutic outcome in inflamed skin therapy. Eur J Pharm Biopharm. 2012;82(1):151-157.

57. Rouvière PE, Li J, Brill DJ, et al. Bionanotechnology application of polypeptides in a hair color product: self-assembly enables expression, processing, and functionality. Biotechnol J. 2013;8(2):247-256.

58. Vogt A, Combadiere B, Hadam S, et al. $40 \mathrm{~nm}$, but not 750 or $1,500 \mathrm{~nm}$, nanoparticles enter epidermal CD1 a+ cells after transcutaneous application on human skin. J Invest Dermatol. 2006;126(6):1316-1322.

59. Rancan F, Gao Q, Graf C, et al. Skin penetration and cellular uptake of amorphous silica nanoparticles with variable size, surface functionalization, and colloidal stability. ACS Nano. 2012;6(8):6829-6842.

60. Mortensen LJ, Oberdörster G, Pentland AP, Delouise LA. In vivo skin penetration of quantum dot nanoparticles in the murine model: the effect of UVR. Nano Lett. 2008;8(9):2779-2787.

61. Mahe B, Vogt A, Liard C, et al. Nanoparticle-based targeting of vaccine compounds to skin antigen-presenting cells by hair follicles and their transport in mice. J Invest Dermatol. 2009;129(5):1156-1164.

62. O’Regan GM, Sandilands A, McLean WH, Irvine AD. Filaggrin in atopic dermatitis. J Allergy Clin Immunol. 2009;124(3 Suppl 2): R2-R6.

63. Yanagisawa R, Takano H, Inoue KI, Koike E, Sadakane K, Ichinose T. Size effects of polystyrene nanoparticles on atopic dermatitislike skin lesions in NC/NGA mice. Int J Immunopathol Pharmacol. 2010; 23(1):131-141.

64. Kirschner N, Poetzl C, von den Driesch P, et al. Alteration of tight junction proteins is an early event in psoriasis: putative involvement of proinflammatory cytokines. Am J Pathol. 2009;175(3):1095-1106.

65. Prow TW, Grice JE, Lin LL, et al. Nanoparticles and microparticles for skin drug delivery. Adv Drug Deliv Rev. 2011;63(6):470-491.

66. Baldo A, Monod M, Mathy A, et al. Mechanisms of skin adherence and invasion by dermatophytes. Mycoses. 2012;55(3):218-223.

67. Baumann L. Skin ageing and its treatment. J Pathol. 2007;211(2): 241-251.

68. Ghadially R, Brown BE, Sequeira-Martin SM, Feingold KR, Elias PM. The aged epidermal permeability barrier. Structural, functional, and lipid biochemical abnormalities in humans and a senescent murine model. J Clin Invest. 1995;95(5):2281-2290.

69. Tezuka T, Qing J, Saheki M, Kusuda S, Takahashi M. Terminal differentiation of facial epidermis of the aged: immunohistochemical studies. Dermatology. 1994;188(1):21-24.

70. Conner SD, Schmid SL. Regulated portals of entry into the cell. Nature. 2003;422(6927):37-44. 
71. Zhang LW, Monteiro-Riviere NA. Mechanisms of quantum dot nanoparticle cellular uptake. Toxicol Sci. 2009;110(1):138-155.

72. Kettiger H, Schipanski A, Wick P, Huwyler J. Engineered nanomaterial uptake and tissue distribution: from cell to organism. Int $J$ Nanomedicine. 2013;8:3255-3269.

73. Luten J, van Nostrum CF, De Smedt SC, Hennink WE. Biodegradable polymers as non-viral carriers for plasmid DNA delivery. $J$ Control Release. 2008;126(2):97-110.

74. Azevedo HS, Reis RL. Understanding the Enzymatic Degradation of Biodegradable Polymers and Strategies to Control their Degradation Rate. Biodegradable Systems in Tissue Engineering and Regenerative Medicine. Boca Raton: CRC Press; 2005:177-201.

75. Mansour AM, Drevs J, Esser N, et al. A new approach for the treatment of malignant melanoma: enhanced antitumor efficacy of an albumin-binding doxorubicin prodrug that is cleaved by matrix metalloproteinase 2 . Cancer Res. 2003;63(14):4062-4066.

76. Yoo HS, Lee EA, Park TG. Doxorubicin-conjugated biodegradable polymeric micelles having acid-cleavable linkages. J Control Release. 2002;82(1):17-27.

77. Weng H, Zhou J, Tang L, Hu Z. Tissue responses to thermallyresponsive hydrogel nanoparticles. J Biomater Sci Polym Ed. 2004;15(9):1167-1180.

78. Küchler S, Radowski MR, Blaschke T, et al. Nanoparticles for skin penetration enhancement - a comparison of a dendritic core-multishellnanotransporter and solid lipid nanoparticles. Eur J Pharm Biopharm. 2009; 71(2):243-250.

79. Küchler S, Abdel-Mottaleb M, Lamprecht A, Radowski MR, Haag R, Schäfer-Korting M. Influence of nanocarrier type and size on skin delivery of hydrophilic agents. Int J Pharm. 2009;377(1-2):169-172.

80. Chiang A, Tudela E, Maibach HI. Percutaneous absorption in diseased skin: an overview. J Appl Toxicol. 2012;32(8):537-563.

81. Hussain Z, Katas H, Mohd Amin MC, Kumolosasi E, Buang F, Sahudin S. Self-assembled polymeric nanoparticles for percutaneous co-delivery of hydrocortisone/hydroxytyrosol: an ex vivo and in vivo study using an NC/Nga mouse model. Int J Pharm. 2013;444(1-2):109-119.

82. Jaques JA, Rezer JF, Ruchel JB, et al. An experimental model of contact dermatitis: evaluation of the oxidative profile of Wistar rats treated with free and nanoencapsulated clobetasol. Redox Rep. 2012;17(5): 206-213

83. Chu CC, Di Meglio P, Nestle FO. Harnessing dendritic cells in inflammatory skin diseases. Semin Immunol. 2011;23(1):28-41.

84. Bak RO, Mikkelsen JG. Regulation of cytokines by small RNAs during skin inflammation. J Biomed Sci. 2010;17:53.

85. Friedman AJ, Han G, Navati MS, et al. Sustained release nitric oxide releasing nanoparticles: characterization of a novel delivery platform based on nitrite containing hydrogel/glass composites. Nitric Oxide. 2008;19(1):12-20.

86. Lansdown AB. Silver. I: Its antibacterial properties and mechanism of action. J Wound Care. 2002;11(4):125-130.

87. Rujitanaroj PO, Pimpha N, Supaphol P. Wound-dressing materials with antibacterial activity from electrospun gelatin fiber mats containing silver nanoparticles. Polymer. 2008;49(21):4723-4732.

88. Shim J, Seok Kang H, Park WS, Han SH, Kim J, Chang IS. Transdermal delivery of mixnoxidil with block copolymer nanoparticles. J Control Release. 2004;97(3):477-484.

89. Knorr F, Lademann J, Patzelt A, Sterry W, Blume-Peytavi U, Vogt A. Follicular transport route - research progress and future perspectives. Eur J Pharm Biopharm. 2009;71(2):173-180.

90. Rancan F, Todorova A, Hadam S, et al. Stability of polylactic acid particles and release of fluorochromes upon topical application on human skin explants. Eur J Pharm Biopharm. 2012;80(1):76-84.
91. Rancan F, Papakostas D, Hadam S, et al. Investigation of polylactic acid (PLA) nanoparticles as drug delivery systems for local dermatotherapy. Pharm Res. 2009;26(8):2027-2036.

92. Cho HK, Cho JH, Choi SW, Cheong IW. Topical delivery of retinol emulsions co-stabilised by PEO-PCL-PEO triblock copolymers: effect of PCL block length. J Microencapsul. 2012;29(8):739-746.

93. Ridolfi DM, Marcato PD, Justo GZ, Cordi L, Machado D, Durán N. Chitosan-solid lipid nanoparticles as carriers for topical delivery of tretinoin. Colloids Surf B Biointerfaces. 2012;93:36-40.

94. Nakamura M, Jo J, Tabata Y, Ishikawa O. Controlled delivery of T-box21 small interfering RNA ameliorates autoimmune alopecia (Alopecia Areata) in a $\mathrm{C} 3 \mathrm{H} / \mathrm{HeJ}$ mouse model. Am J Pathol. 2008;172(3):650-658.

95. Romero EL, Morilla MJ. Highly deformable and highly fluid vesicles as potential drug delivery systems: theoretical and practical considerations. Int J Nanomedicine. 2013;8:3171-3186.

96. Zampieri AL, Ferreira FS, Resende EC, et al. Biodegradable polymeric nanocapsules based on poly(DL-lactide) for genistein topical delivery: obtention, characterization and skin permeation studies. J Biomed Nanotechnol. 2013;9(3):527-534.

97. Venuganti VV, Perumal OP. Effect of poly(amidoamine) (PAMAM) dendrimer on skin permeation of 5-fluorouracil.Int J Pharm. 2008; 361(1-2):230-238.

98. Zhao QH, Zhang Y, Liu Y, et al. Anticancer effect of realgar nanoparticles on mouse melanoma skin cancer in vivo via transdermal drug delivery. Med Oncol. 2010;27(2):203-212.

99. Casas A, Battah S, Di Venosa G, et al. Sustained and efficient porphyrin generation in vivo using dendrimer conjugates of 5-ALA for photodynamic therapy. J Control Release. 2009;135(2): 136-143.

100. Reddy ST, Swartz MA, Hubbell JA. Targeting dendritic cells with biomaterials: developing the next generation of vaccines. Trends Immunol. 2006;27(12):573-579.

101. Duffy D, Perrin H, Abadie V, et al. Neutrophils transport antigen from the dermis to the bone marrow, initiating a source of memory CD8+ T cells. Immunity. 2012;37(5):917-929.

102. Liard C, Munier S, Arias M, et al. Targeting of HIV-p24 particlebased vaccine into differential skin layers induces distinct arms of the immune responses. Vaccine. 2011;29(37):6379-6391.

103. Hansen S, Lehr CM. Nanoparticles for transcutaneous vaccination. Microb Biotechnol. 2012;5(2):156-167.

104. Matsuo K, Hirobe S, Okada N, Nakagawa S. Frontiers of transcutaneous vaccination systems: novel technologies and devices for vaccine delivery. Vaccine. 2013;31(19):2403-2415.

105. Li J, Li X, Zhang Y, et al. Gene therapy for psoriasis in the K14VEGF transgenic mouse model by topical transdermal delivery of interleukin-4 using ultradeformable cationic liposome. J Gene Med. 2010;12(6):481-490.

106. Özbaş-Turan S, Akbuğa J. Plasmid DNA-loaded chitosan/TPP nanoparticles for topical gene delivery. Drug Deliv. 2011;18(3): 215-222.

107. Aissaoui A, Chami M, Hussein M, Miller AD. Efficient topical delivery of plasmid DNA to lung in vivo mediated by putative triggered, PEGylated pDNA nanoparticles. J Control Release. 2011;154(3): 275-284.

108. Zheng D, Giljohann DA, Chen DL, et al. Topical delivery of siRNA-based spherical nucleic acid nanoparticle conjugates for gene regulation. Proc Natl Acad Sci U S A. 2012;109(30):11975-11980.

109. Segura T. Formulations and Delivery Limitations of NucleicAcid-Based Therapies. Handbook of Pharmaceutical Biotechnology. 2010;1013-1059 


\section{Publish your work in this journal}

Clinical, Cosmetic and Investigational Dermatology is an international, peer-reviewed, open access, online journal that focuses on the latest clinical and experimental research in all aspects of skin disease and cosmetic interventions. All areas of dermatology will be covered; contributions will be welcomed from all clinicians and

basic science researchers globally. This journal is indexed on CAS.

The manuscript management system is completely online and includes a very quick and fair peer-review system, which is all easy to use. Visit http://www.dovepress.com/testimonials.php to read real quotes from published authors.

Submit your manuscript here: http://www.dovepress.com/clinical-cosmetic-and-investigational-dermatology-journal 